\title{
ACÚMULO DE MICRONUTRIENTES EM COLMOS E PALHADA DE CINCO CULTIVARES DE CANA-DE-AÇÚCAR DE CICLO PRECOCE
}

\author{
TASSO JÚNIOR, Luiz Carlos ${ }^{1}$ \\ SILVA NETO, Hélio Francisco da ${ }^{2}$ \\ SILVA, Joana Diniz Rosa ${ }^{3}$ \\ MARQUES, Marcos Omir ${ }^{4}$ \\ CAMILOTTI, Fábio ${ }^{5}$
}

Recebido em: 2010-12-17 Aprovado em: 2011-03-23

ISSUE DOI: $10.3738 / 1982.2278 .537$

RESUMO: Objetivando quantificar o acúmulo de micronutrientes em cultivares de maturação precoce de cana-de-açúcar durante o ciclo da cultura, com intuito de se ter subsidios para o manejo de fertilizantes, instalouse, na fazenda Angico Preto município de Colina, SP, um experimento com cinco cultivares precoces (RB855453; RB855156; RB835486; SP89-1115 e IAC91-2195), visando determinar o acúmulo de cobre, ferro, manganês e zinco nos colmos e palhada. O experimento foi conduzido em uma área de Latossolo, com delineamento experimental em blocos ao acaso com cinco tratamentos e quatro repetições, onde se determinou a produtividade de colmos industrializáveis e de palhada. Nos resultados obtidos, destacaram-se os cultivares SP 89-1115 (cobre e zinco) e RB855156 (Manganês e zinco), sugerindo a realização de investigações a respeito da disponibilidade desses elementos no solo em que esses cultivares estão estabelecidos e a capacidade de absorção desses nutrientes pelos cultivares estudados.

Palavras-chave: Colmos. Exportação. Fertilizantes. Nutrição. Saccharum spp.

\section{TRACE NUTRIENTS ACCUMULATION IN STALKS AND DRY LEAFS FROM PRECOCIOUS SUGAR CANE CULTIVARS}

SUMMARY: The experiment was conducted out with the objective to evaluate the trace nutrients accumulation in precocious sugar cane cultivars, during the 2005/2006 crop season. The experimental area was localized in Angico Preto County, State of São Paulo, Brasil, on an Oxisol. The treatments were five sugarcane cultivars (RB855453; RB855156; RB835486; SP89-1115 e IAC91-2195). The experimental design was a randomized blocks with four replications. The variables analyzed was stalks copper, manganese and zinc contents. According to results, the highlights sugarcane cultivars were SP89-1115 (copper and zinc) and RB855156 (manganese and zinc) suggesting these soil elements availability and these elements accumulation capacity by these cultivars.

Keywords: Export. Fertilizers. Nutrition. Saccharum spp. Stems.

\footnotetext{
${ }^{1}$ Bolsista CAPES - Pós-doutorando em Produção Vegetal pela Faculdade de Ciências Agrárias e Veterinárias FCAV - UNESP/Jaboticabal. E-mail: lctasso@yahoo.com.br

${ }^{2}$ Bolsista CNPq - Doutorando em Produção Vegetal pela Faculdade de Ciências Agrárias e Veterinárias FCAV UNESP/Jaboticabal. E-mail: heliofsn@hotmail.com

${ }^{3}$ Bolsista CAPES - Mestranda em Produção Vegetal pela Faculdade de Ciências Agrárias e Veterinárias FCAV UNESP/Jaboticabal. E-mail: jodiniz2@yahoo.com.br

${ }^{4}$ Professor Adjunto do Departamento de Tecnologia da Faculdade de Ciências Agrárias e Veterinárias FCAV UNESP/Jaboticabal. E-mail: omir@ fcav.unesp.br

${ }^{5}$ Professor da Faculdade de Tecnologia de Jaboticabal. Fatec-Jaboticabal. E-mail: fabiocamilotti@hotmail.com
} 


\section{INTRODUÇÃO}

Nos últimos anos, o Brasil vem obtendo aumentos significativos em sua participação mundial na produção de cana-de-açúcar e seus derivados. Considerada um dos produtos de maior competitividade no cenário do agronegócio, a cana-de-açúcar representa $8 \%$ do Produto Interno Bruto (PIB) agrícola nacional e 35\% do PIB agrícola do Estado de São Paulo, que atualmente responde por aproximadamente $60 \%$ da colheita brasileira. A atividade é responsável por cerca de um milhão de empregos diretos, dos quais 511 mil apenas na produção de cana-de-açúcar. O restante está distribuído na agroindústria de açúcar, de álcool e outros relacionados a esta cultura. São Paulo, isoladamente, reúne 400 mil empregos diretos no setor (ANUÁRIO BRASILEIRO DA CANA-DE-AÇÚCAR, 2005).

No acumulado da safra 2008/2009, o volume de cana processada registrado até o ultimo dia de dezembro na região Centro-Sul chegou a 497,7 milhões de toneladas das quais $40 \%$ deste volume foi destinado à fabricação de açúcar e $60 \%$ destinado à produção de álcool. O Estado de São Paulo contribuiu com um volume de 341,8 milhões de toneladas que corresponde a $69 \%$ do total da cana esmagada na região Centro-Sul (UNICA, 2009).

O desenvolvimento da cana-de-açúcar tem raízes históricas, que abrange principalmente a busca de tecnologias adequadas, dentro de um contexto da evolução do sistema produtivo, compreendendo aspectos técnicos, biológicos, econômicos e sociais (VASCONCELOS, 1998).

Para o aumento da produtividade agrícola, a busca de novos cultivares assume um papel imprescindível. Neste sentido há necessidade de trabalhos de pesquisas intensivos abordando propriedades agroindustriais, tais como resistência às doenças, capacidade de brotação, elevados números de $\operatorname{ATR}(\mathrm{s})$, alta produtividade e aspectos relacionados às condições de clima e solo (TASSO JÚNIOR, 2007).

Os macro e micronutrientes exercem funções específicas na vida da planta, embora em algumas delas possa haver, dependendo do elemento, um certo grau de substituição (MALAVOLTA; VITTI; OLIVEIRA, 1989). Tais funções podem ser classificadas em três grandes grupos: Estrutural, Constituinte de enzima e Ativador enzimático.

O cobre é absorvido pelas raízes na forma de $\mathrm{Cu}^{+2}$ e também na forma quelato com EDTA e, embora tenha lugar a absorção por difusão ou fluxo massa, a sua mobilidade faz com que seu suprimento às plantas seja feito em maior proporção por interceptação radicular (OLIVER ; BARBIERI, 1966; JARVIS, 1981 citados por CASAGRANDE, 1991).

$\mathrm{Na}$ exportação de $\mathrm{Cu}$ para o colmo, Malavolta (1994) obteve resultados de $190 \mathrm{~g}$ de $\mathrm{Cu}$ 
$100 \mathrm{TCI}^{-1}$. No entanto, Soriano (2007) e Franco et al. (2008) obtiveram menores valores, $40 \mathrm{~g}$ de $\mathrm{Cu} .100 \mathrm{TCI}^{-1}$ e $44 \mathrm{~g}$ de $\mathrm{Cu} .100 \mathrm{TCI}^{-1}$, respectivamente.

O elemento ferro na planta atua como ativador de enzimas como Heme-Perofidase, catalase, oxidase de sulfito, além de atuar na fotossíntese, respiração, fixação biológica de nitrogênio e assimilação de nitrogênio e enxofre (MALAVOLTA; VITTI; OLIVEIRA, 1989).

De acordo com Malavolta (1994) a extração de $\mathrm{Fe} .100 \mathrm{TCI}^{-1}$ foi de 3.700 g. Soriano (2007) e Vitti; Oliveira; Quintino apud Segato et al. (2006) obtiveram exportação da ordem de

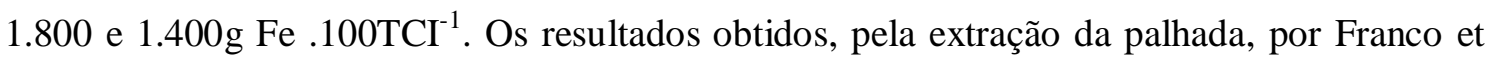
al. (2008), foi de $1116 \mathrm{~g}$ de Fe .100TCI ${ }^{-1}$.

O Mn é absorvido por difusão. Na planta, funciona como ativador de varias enzimas, como sintetize de glutationa, metionina; AT passe, quinase pirúvica, enolose, desidrogenase isocitrica, descarboxilase pirúvica e pirofosforilase (MALAVOLTA; VITTI; OLIVEIRA, 1989). Vitti; Oliveira; Quintino apud Segato et al. (2006) e Franco et al. (2008) apresentaram resultados semelhantes de extração, na ordem de $2.470 \mathrm{~g}$ e $2.420 \mathrm{~g}$ de $\mathrm{Mn}^{2} .100 \mathrm{TCI}^{-1}$. Por outro lado, Orlando Filho et al. (1980) e Malavolta (1994) apresentaram valores semelhantes de $1.188 \mathrm{~g}$ e $1.110 \mathrm{~g}$ de $\mathrm{Mn} .100 \mathrm{TCI}^{-1}$, respectivamente.

Os resultados obtidos por Franco et al. (2008), para palha, foram de $309 \mathrm{~g}$ e $108 \mathrm{~g}$ de Mn .100TCI ${ }^{-1}$, com exceção do cultivar RB855156.

O zinco potencializa a produção do hormônio de crescimento (auxina)-sintetase do triptofano e indiretamente pela síntese de proteínas com evidencias de que este nutriente atua no metabolismo do nitrogênio (MALAVOLTA; VITTI; OLIVEIRA, 1997). Este elemento se concentra nas zonas de crescimento devido à maior concentração auxínica.

Orlando Filho (1993) encontrou no colmo e na folha valores de 369 e 223 g de Zn para a produção de $100 \mathrm{t}$ de colmos $\mathrm{CI}^{-1}$

A deficiência de cobre nas plantas pode ser diagnosticada visualmente, pelos seguintes sintomas: pequeno desenvolvimento da planta, folhas cloróticas e difícil aparecimento de folhas novas, as folhas se curvam em direção ao solo (droopy top). Em casos mais agudos ocorre clorose foliar dividida em pequenos retângulos (VITTI; OLIVEIRA; QUINTINO apud SEGATO et al., 2006).

Segundo Tokeshi (1991) citado por Vitti et al. (2006). Os sintomas de deficiência de ferro em cana-de-açúcar podem ocorrer em cana-planta ou soca, com enraizamento superficial no período em que os brotos estão sendo alimentados pelas raízes das soca ou do colmo mãe (tolete).

A deficiência de manganês ocorre principalmente em solos alcalinos, devido à 
presença de rochas calcarias, conchas marinhas ou com calagem excessiva (TOKESHI 1991 citado por VITTI et al., 2006).

Em cana-de-açúcar, as mudas provenientes e plantadas em solos deficientes em zinco, ao germinarem dão origem a plantas com pequenos alongamentos do palmito, com tendência das folhas saírem todas do vértice foliar na mesma altura, formando o sintoma de "leque" (TOKESHI, 1991, citado por (VITTI; OLIVEIRA; QUINTINO apud SEGATO et al., 2006).

De cada 100 reais contabilizados como custo operacional de produção de cana, mais de 20 reais são atribuídos aos insumos agrícolas e, $70 \%$ do gasto com insumos, são destinados aos fertilizantes. Portanto, conhecer as exigências minerais que cada cultivar necessita, é de suma importância, para diminuir custos na implantação e condução da lavoura, como forma de evitar problemas advindos de uma aplicação de fertilizantes em excesso.

Assim o objetivo do presente trabalho foi identificar o comportamento dos cinco cultivares de ciclo precoce estudado em relação ao acúmulo de micronutrientes nos colmos e na palhada.

\section{MATERIAL E MÉTODO}

A instalação do Experimento ocorreu na fazenda Angico Preto, município de Colina, SP, com Latitude $20^{\circ} 25^{\prime}$ e Longitude $48^{\circ} 19^{\prime}$, situada no centro norte do Estado de São Paulo, altitude média de $590 \mathrm{~m}$. acima do nível do mar, relevo caracterizado como suave ondulado e, com precipitação média no período de 1997 a 2005 de 1432 mm/ano.

Através de amostras retiradas para caracterização física e química do solo, nas profundidades de 0-20; 20-40 e 80-100, apresentadas nas tabelas 1 e 2 respectivamente, o mesmo foi classificado como Latossolo Vermelho-Amarelo eutrófico típico (EMBRAPA, 1999).

Tabela 1. Dados da análise granulométricas do solo.

\begin{tabular}{ccccc}
\hline $\begin{array}{c}\text { Profundidade } \\
(\mathrm{cm})\end{array}$ & Argila & Silte & Areia Fina & Areia Grossa \\
$\%$ & $\%$ & $\%$ & $\%$ & $\%$ \\
\hline $0-20$ & 16,2 & 4,75 & 32,60 & 46,50 \\
$20-40$ & 20,9 & 3,09 & 36,20 & 39,81 \\
$80-100$ & 21,12 & 3,70 & 36,00 & 39,18 \\
\hline
\end{tabular}

Fonte: Coopercana. Setembro de 2002 de acordo com o sistema IAC. 
Foi estudado os cultivares com ciclo de maturação precoce, sendo eles o RB855453; RB855156; RB835486; SP89-1115 e IAC91-2195. Para padronizar a origem das mudas, idade e as condições edafoclimáticas para seu desenvolvimento e formação realizaram-se na mesma área, no ano de 2003, o plantio dos cultivares a serem testados.

O plantio da área experimental ocorreu em fevereiro de 2004. Realizou-se o delineamento experimental em blocos ao acaso com 5 tratamentos e 4 repetições. Cada parcela foi constituída por cinco linhas de oito metros de comprimento espaçadas de 1,5m, resultando em uma área total de $60 \mathrm{~m}^{2}$ para cada parcela.

Tabela 2. Dados da análise química do solo da área experimental.

\begin{tabular}{ccccccccccc}
\hline $\begin{array}{c}\text { Prof. } \\
\mathrm{cm}\end{array}$ & $\begin{array}{c}\mathrm{Ph} \\
\mathrm{CaCl}_{2}\end{array}$ & $\mathrm{MO}$ & $\begin{array}{c}\mathrm{P} \\
\mathrm{resina}\end{array}$ & $\mathrm{K}$ & $\mathrm{Ca}$ & $\mathrm{Mg}$ & $\mathrm{H}+\mathrm{Al}$ & $\mathrm{SB}$ & $\mathrm{CTC}$ & $\mathrm{V}$ \\
\hline & & $\mathrm{g} \mathrm{dm}^{-3}$ & $\mathrm{mg} \mathrm{dm}^{-3}$ & -10 & & & & & & \\
\hline $0-20$ & 5,9 & 16 & 7 & 2,2 & 28 & 13 & 16 & 43,2 & 59,2 & 73 \\
$20-40$ & 5,2 & 11 & 5 & 1,2 & 12 & 6 & 20 & 19,2 & 39,2 & 49 \\
$80-100$ & 4,8 & 9 & 2 & 0,4 & 7 & 3 & 12 & 10,4 & 28,4 & 37 \\
\hline
\end{tabular}

Fonte: Coopercana. Setembro de 2002 de acordo com o sistema IAC.

Para determinar a exportação dos micronutrientes e da massa verde produzida pelos cultivares de cana-de-açúcar, adotaram-se amostragens retiradas nas três linhas centrais da parcela. Portanto, ao longo de 3,5 m procedeu-se à coleta de 6 colmos $(50 \mathrm{~cm}$ de linha de cana) seguidos, sendo que este mesmo procedimento foi executado em 4 repetições. $\mathrm{Na}$ determinação da massa verde produzida, foi separada a palhada (folhas secas, folhas verdes e ponteiro na altura do desponte) dos colmos industrializáveis. Para a determinação da massa verde produzida por hectare, utilizaram-se os resultados de massa do material obtido na área amostrada $\left(0,50 \mathrm{~m}\right.$ x 1,5 m) e extrapolou-se os resultados obtidos para $10.000 \mathrm{~m}^{2}$.

Para a determinação dos micronutrientes, foram realizadas as análises químicas aos 16 meses após o plantio, considerando os colmos e a palhada como material que seriam analisados.

O material coletado foi lavado em água corrente, água destilada e a água desionizada. Em seguida procedeu-se à desintegração do material em desintegrador do tipo forrageiro. Após homogeneização, uma sub-amostra de aproximadamente $100 \mathrm{~g}$ foi mantida em estufa com circulação forçada de ar $\left(60-70^{\circ} \mathrm{C}\right)$, até que se atingisse o peso constante. Após a secagem, o material foi submetido à moagem em moinho tipo Willey. Do material obtido, 
pesou-se $0,5 \mathrm{~g}$ desse material e submeteu-o à digestão nitricoperclórica e $0,2 \mathrm{~g}$ do material moído para a digestão sulfúrica.

\section{RESULTADOS E DISCUSSÃO}

As produtividades determinadas por TCH (tonelada de colmo por ha) e TPH (tonelada de palha por ha) para cada cultivar são apresentadas na Tabela 3.

As avaliações de produtividades apresentaram diferenças significativas, sendo o cultivar RB855453 o mais produtivo e seguido pelos cultivares RB835486, IAC91-2195 e RB855156 com produtividades intermediarias. O cultivar SP89-1115 apresentou resultados inferiores aos demais, sendo este o único cultivar testado oriundo de cultura de meristema.

Os cultivares que apresentaram as maiores produtividades de colmos também foram os que produziram as maiores quantidades de palha. Esta relação também foi observada nos cultivares com baixa produtividade, que possuíram baixa produção de palha.

Observam-se também diferenças nos valores de TPH. Sendo os cultivares RB855453 e RB835486 os de maiores resultados, seguidos por RB855156 e IAC91-2195 com valores intermediários. O cultivar SP89-1115 apresentou os menores resultados (Tabela 3).

Tabela 3. Produtividade agrícola e de palhada para os cinco cultivares de ciclo de maturação precoce testados.

\begin{tabular}{lcc} 
Cultivares & TCH & TPH \\
RB855453 & $165,55 \mathrm{~A}^{1}$ & $11,78 \mathrm{~A}$ \\
RB855156 & $124,77 \mathrm{C}$ & $8,07 \mathrm{~B}$ \\
RB835486 & $138,61 \mathrm{~B}$ & $10,67 \mathrm{~A}$ \\
SP89-1115 & $111,11 \mathrm{D}$ & $3,32 \mathrm{C}$ \\
IAC91-2195 & $132,22 \mathrm{~B}$ & $6,91 \mathrm{~B}$ \\
\hline Teste F. & $54,78^{* * 2}$ & $39,75^{* *}$ \\
C.V. & 63,67 & 39,42
\end{tabular}

${ }^{\top}$ Números seguidos pela mesma letra não diferem significativamente entre si pelo Teste de Tukey a 5\%.** significativo a $1 \%$ de probabilidade

As Tabelas 4 e 5 apresentam os valores médios das extrações de micronutrientes do solo, pelos cultivares de cana-de-açúcar estudados.

O nutriente mais extraído do solo e exportado para o colmo e para a palha foi o Fe. O menos exportado foi o cobre. 
Tabela 4. Teores de micronutrientes extraídos pelos colmos de cinco cultivares de ciclo precoce de cana-de-açúcar.

\begin{tabular}{|c|c|c|c|c|}
\hline Cultivares & $\mathrm{Cu}$ & $\mathrm{Fe}$ & $\mathrm{Mn}$ & $\mathrm{Zn}$ \\
\hline & \multicolumn{4}{|c|}{$\mathrm{g}$ por $100 \mathrm{TCI}$} \\
\hline IAC91-2195 & $145 \mathrm{~B}^{1}$ & $2796 \mathrm{C}$ & $1999 \mathrm{C}$ & $385 \mathrm{C}$ \\
\hline SP89-1115 & $180 \mathrm{~A}$ & $2742 \mathrm{C}$ & $2207 \mathrm{~B}$ & $555 \mathrm{~A}$ \\
\hline RB855453 & $78 \mathrm{C}$ & $3070 \mathrm{~B}$ & $2900 \mathrm{~A}$ & $245 \mathrm{D}$ \\
\hline RB855156 & $149 \mathrm{~B}$ & $2692 \mathrm{C}$ & $2988 \mathrm{~A}$ & $535 \mathrm{~A}$ \\
\hline RB835486 & $58 \mathrm{D}$ & $4365 \mathrm{~A}$ & $1513 \mathrm{D}$ & $439 \mathrm{~B}$ \\
\hline Teste $\mathrm{F}$ & $23,77 * *^{2}$ & $6,13 * *$ & $7,32 * *$ & $35,65 * *$ \\
\hline $\mathrm{CV}$ & 14,70 & 8,23 & 15,92 & 25,15 \\
\hline
\end{tabular}

Tabela 5. Teores de micronutrientes extraídos pela palhada de cinco cultivares de ciclo precoce de cana-de-açúcar.

\begin{tabular}{|c|c|c|c|c|}
\hline Cultivares & $\mathrm{Cu}$ & $\mathrm{Fe}$ & $\mathrm{Mn}$ & $\mathrm{Zn}$ \\
\hline \multicolumn{5}{|c|}{ g por $100 \mathrm{TCI}$} \\
\hline IAC91-2195 & $7,08 \mathrm{C}$ & $871 \mathrm{C}$ & $699 \mathrm{~A}$ & $106 \mathrm{~A}$ \\
\hline SP89-1115 & $11,83 \mathrm{~A}$ & $1254 \mathrm{~A}$ & $451 \mathrm{~B}$ & $85 \mathrm{~B}$ \\
\hline RB855453 & $10,90 \mathrm{~B}$ & $953 \mathrm{~B}$ & $478 \mathrm{~B}$ & $48 \mathrm{C}$ \\
\hline RB855156 & $3,74 \mathrm{D}$ & $318 \mathrm{E}$ & $231 \mathrm{C}$ & $1,3 \mathrm{D}$ \\
\hline RB835486 & $10,64 \mathrm{~B}$ & $789 \mathrm{D}$ & $464 \mathrm{~B}$ & $42 \mathrm{C}$ \\
\hline Teste F & $4,04 * *$ & $11,11 * *$ & $6,01 * *$ & $16,18 * *$ \\
\hline $\mathrm{CV}$ & 18,11 & 11,46 & 14,08 & 16,73 \\
\hline
\end{tabular}

As extrações de Fe pelos colmos oscilaram de $2.692 \mathrm{~g}$ a $4.365 \mathrm{~g}$ de $\mathrm{Fe}^{100 \mathrm{TCI}^{-1}}$, sendo a maior extração apresentada pelo cultivar RB835486 e a menor extração pelo cultivar RB855156. De acordo com Malavolta (1994) a extração de $\mathrm{Fe} .100 \mathrm{TCI}^{-1}$ é de $3.700 \mathrm{~g}$. Soriano (2007), Vitti; Oliveira; Quintino apud Segato et al. (2006) e Orlando Filho (1993) obtiveram exportação na ordem de $1.800 \mathrm{~g}, 1.400 \mathrm{~g}$ e $1.393 \mathrm{~g} \mathrm{Fe} .100 \mathrm{TCI}^{-1}$ respectivamente. Os resultados obtidos para extração da palhada oscilaram de $318 \mathrm{~g}$ a $1.254 \mathrm{~g}$ de $\mathrm{Fe} .100 \mathrm{TCI}^{-1}$, sendo que quatro das cinco cultivares obtiveram valores próximo aos valores apresentados por Franco et al. (2008) que foi de $1116 \mathrm{~g}$ de Fe.100TCI ${ }^{-1}$.

Em relação ao Mn os cultivares apresentaram diferenças de acúmulo, com destaque para RB855156 e RB855453 com 2988 e 2900 g de Mn.100TCI ${ }^{-1}$. Comparando os resultados 
obtidos, observou-se que Vitti; Oliveira; Quintino apud Segato et al. (2006) e Franco et al. (2008) apresentaram resultados semelhantes de extração em relação a esse experimento, na

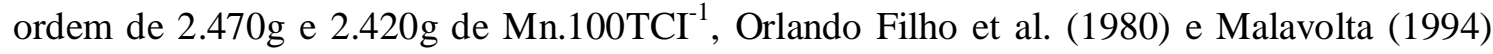
apresentaram valores semelhantes de $1.188 \mathrm{~g}$ e $1.110 \mathrm{~g}$ de $\mathrm{Mn}^{100 \mathrm{TCI}^{-1}}$ respectivamente.

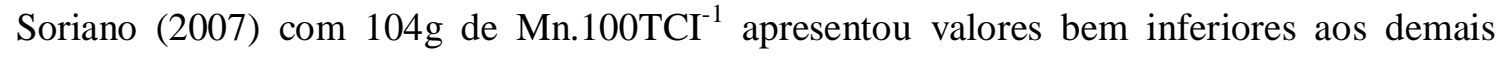
autores. Com base nesses resultados, podemos concluir que os mesmos ficaram próximos aos valores dos trabalhos encontrados na literatura. Em relação de teores na palhada, o cultivar IAC91-2195 com 699g de Mn.100TCI ${ }^{-1}$ se destacou dos demais. O cultivar RB855156 com $231 \mathrm{~g}$ de $\mathrm{Mn} 100 \mathrm{TCI}^{-1}$ foi a que menos exportou manganês para a palhada. Os resultados ficaram acima dos obtidos por Franco et al. (2008) que foram de $309 \mathrm{~g}$ e $108 \mathrm{~g}$ de $\mathrm{M}^{1}$.100TCI$^{-1}$, com exceção do cultivar RB855156.

Na avaliação do acúmulo de zinco no colmo, os cultivares em destaque foram SP891115 com $555 \mathrm{~g}$ de $\mathrm{Zn}$. 00TCI ${ }^{-1}$ e RB855156 com 535 g de Zn. 100TCI ${ }^{-1}$. Na posição intermediária aparece o RB835486 com 439 g de Zn.100TCI ${ }^{-1}$ e nas inferiores IAC91-2195 com 385 g de $\mathrm{Zn}$.100TCI ${ }^{-1}$ e RB855453 com 245 g de Zn.100TCI ${ }^{-1}$. Para os dados de acumulo na palhada, os que se destacaram foram IAC91-2195 com 106g de Zn .100TCI ${ }^{-1}$ e SP89-1115 com $85 \mathrm{~g}$ de Zn.100TCI ${ }^{-1}$. As intermediarias foram RB835486 com 42g de Zn.100TCI ${ }^{-1}$ e RB855453 com 48g de Zn.100TCI ${ }^{-1}$. Na posição inferior tem-se o RB855156 com 1,25 g de Zn.100 $\mathrm{TCI}^{-1}$.

Orlando Filho (1993) encontrou, no colmo e na folha, valores de 369 e 223 g de $\mathrm{Zn}$ para a produção de $100 \mathrm{t}$ de colmos $\mathrm{CI}^{-1}$.

O nutriente mais extraído do solo e exportado para o colmo e para a palhada foi o Fe. O menos exportado foi o cobre.

Os cultivares que apresentaram as maiores produtividades de colmos foram os que produziram as maiores quantidades de palhada. Esta relação também foi observada nos cultivares com baixa produtividade, que possuíram uma baixa produção de palhada.

Uma maior produção de colmos e palhada não se relacionaram a uma maior extração de micronutriente.

\section{REFERÊNCIAS}

ANUÁRIO BRASILEIRO DA CANA-DE-AÇÚCAR Santa Cruz do Sul: Gazeta Santa Cruz, 2005. 136p.

CASAGRANDE, A. A. Tópicos de morfologia e fisiologia da cana-de-açúcar. Jaboticabal: FUNEP, 1991, 157p. 
FRANCO, H.C.J.et al. Acúmulo de Nutrientes pela Cana-Planta. STAB: Açúcar, Álcool e Subprodutos, Piracicaba, v. 26, n.5, p. 41-45, maio/jun. 2008.

EMBRAPA - Empresa Brasileira de Pesquisa Agropecuária. Centro Nacional de Pesquisa de Solos. Sistema Brasileiro de Classificação de Solos. Brasília: Embrapa Produção e Informação, 1999. 412p.

JARVIS, S. C. Copper concentrations in plant and their relationship to soil properties. In: LONERAGAN, J. F.; ROBISON, A. D.; GRAHAN, R. D. Copper in Soil and plants. London: American Press, 1981 p. 265-285.

MALAVOLTA, E.; VITTI, G.C.; OLIVEIRA, S. A. Avaliação do estado nutricional das plantas: princípios e aplicações. 2 ed. Piracicaba: POTAFÓS, 1997- 319p.

MALAVOLTA, E. Nutrient and fertilizer management in sugarcane. Basel: International Potash Institute, 1994. 104p.

MALAVOLTA, E.; VITTI, G. C.; OLIVEIRA, S. A. Avaliação do estado nutricional das plantas: princípios e aplicações. Piracicaba: Associação Brasileira para a Pesquisa da Potassa e do Fosfato, 1989. 201p.

ORLANDO FILHO, J. Calagem e adubação da cana-de-açúcar. In: CÂMARA, G.M.S. ; OLIVEIRA, E.A.M. (eds). Produção de cana-de-açúcar. Piracicaba: FEALQ/USP, 1993. p.133-146.

OLIVER, S.; BARBER, S. A. Mechanisms for the movement of $\mathrm{Mn}, \mathrm{Fe}, \mathrm{B}, \mathrm{Cu}, \mathrm{Zn}, \mathrm{Al}$ and $\mathrm{Si}$ from the soil to the surface of soybean roots. Soil Sci. Soc. Am. Proc., n.30, p. 468-470, 1966.

ORLANDO FILHO, J.; ZAMBELLO JUNIOR, E.; HAAG, H. Acumulação de manganês pela parte aérea da cana-de-açúcar em função da idade. Boletim Técnico Planalsucar, Piracicaba, v.2, n.2, p. 3-30, 1980.

SORIANO, H.L. Extração e eficiência de macro e micronutrientes em oito cultivares RB de cana-de-açúcar. 2007. 19 f. Trabalho de Conclusão de Curso (Graduação em Agronomia) - Centro de Ciências Agrárias, Universidade Federal de Alagoas, Rio Largo.

TASSO JUNIOR, L. C. Caracterização Agrotecnológica de Cultivares de Cana-deAçúcar (Saccharum spp.) na Região Centro-Norte do Estado de São Paulo. 2007. 167f. Tese (Doutorado) - Universidade Estadual Paulista, Faculdade de Ciências Agrárias e Veterinárias. Jaboticabal.

TOKESHI, H.; Cana-de-açúcar. In: FERREIRA, M. E.; CRUZ, M. C. P. (orgs.). Micronutrientes na agricultura. Piracicaba: Potafos/CNPq, 199, p. 102-144.

ÚNICA. Acompanhamento de safra 2008/2009 - Assessoria técnica. São Paulo: ÚNICA, 2008. 11p.

VASCONCELOS, A.C.M. Comportamento de clones IAC e variedades de cana-deaçúcar (Saccharum spp) nas condições edafoclimáticas da região do Vale do Paranapanema. 1998. 108f. Dissertação (Mestrado em Produção Vegetal). Faculdade de Ciências Agrárias e Veterinárias, Universidade Estadual Paulista, Jaboticabal. 
VITTI, G.C.; OLIVEIRA, D.B.de ; QUINTINO, T.A. Micronutrientes na cultura da cana-deaçúcar. In: SEGATO, S.V.et al. (Org.). Atualização em produção de cana-de-açúcar.

Piracicaba: CERES, 2006, p. 121-138. 